\title{
Visual outcome after fractionated stereotactic radiation therapy of benign anterior skull base tumors
}

\author{
Arnar Astradsson - Anne Katrine Wiencke • \\ Per Munck af Rosenschold • Svend-Aage Engelholm • \\ Lars Ohlhues $\cdot$ Henrik Roed $\cdot$ Marianne Juhler
}

Received: 20 July 2013/Accepted: 31 January 2014/Published online: 15 February 2014

(C) The Author(s) 2014. This article is published with open access at Springerlink.com

\begin{abstract}
To determine visual outcome including the occurrence of radiation induced optic neuropathy (RION) as well as tumor control after fractionated stereotactic radiation therapy (FSRT) of benign anterior skull base meningiomas or pituitary adenomas. Thirty-nine patients treated with FSRT for anterior skull base meningiomas and 55 patients treated with FSRT for pituitary adenomas between January 1999 and December 2009 with at least 2 years follow-up were included. Patients were followed up prospectively with magnetic resonance imaging scans, visual acuity and visual field examinations. RION was found in four $(10 \%)$ patients with anterior skull base meningiomas and seven patients $(13 \%)$ with pituitary adenomas. The five-year actuarial freedom from $25 \%$ RION visual field loss was $94 \%$ following FSRT. Actuarial 2-, 5- and 10-year tumor control rates were 100, 88.4 and $64.5 \%$ for anterior skull base meningiomas and 100, 98.2 and $94.9 \%$ for pituitary adenomas, respectively. Patients with an impaired visual field function pre-FSRT
\end{abstract}

A. Astradsson $(\bowtie) \cdot$ M. Juhler

Department of Neurosurgery, Rigshospitalet, Copenhagen, Denmark

e-mail: arnar.astradsson@gmail.com

\section{A. K. Wiencke}

Department of Ophthalmology, Rigshospitalet, Copenhagen, Denmark

\author{
A. K. Wiencke \\ Department of Ophthalmology, Glostrup Hospital, Copenhagen, \\ Denmark \\ P. Munck af Rosenschold - S.-A. Engelholm - L. Ohlhues · \\ H. Roed \\ Department of Radiation Oncology, Finsen Center, \\ Rigshospitalet, Copenhagen, Denmark
}

were more likely to experience worsened function $(p=0.016)$. We found that RION, was a relatively uncommon event, in a large prospective cohort of patients that were systematically monitored following FSRT of benign anterior skull base tumors. Long term tumor control was favorable, especially for pituitary adenomas.

Keywords Fractionated stereotactic radiation therapy · Visual outcome - Radiation induced optic neuropathy . Tumor control - Anterior skull base meningiomas . Pituitary adenomas

\section{Introduction}

Stereotactic radiosurgery (SRS) and fractionated stereotactic radiotherapy (FSRT) are image-guided highly precise irradiation therapies used for tumor tissue or malformations, with the aim of maximizing the irradiation dose to the target and minimizing the dose to nearby normal tissue structures [1]. SRS/FSRT is the treatment of choice if open surgery is considered too risky; as an adjuvant treatment to surgery; when complete surgical resection is not possible or with residual or recurrent disease [2]. Also, in the case of hormone secreting pituitary tumors, FSRT may be indicated to treat hypersecretion [1]. Accordingly, the most frequent indications for FSRT or SRS in the anterior skull base are meningiomas of the cavernous sinus and pituitary tumors with parasellar extension due to risk of cranial nerve injury and non-radicality with surgery for such lesions [3]. When choosing this treatment modality for lesions close to eloquent brain structures, it may be feasible to choose the fractionated therapy (FSRT), which combines the high accuracy of stereotactic radiation with the biological advantage of 
fractionation [1, 2]. Few long-term reports on visual complications after FSRT for anterior skull base tumors exist [4]. Furthermore, studies correlating irradiation dose to the optic pathways are few. We here describe visual outcome and the occurrence of radiation induced optic neuropathy (RION) and tumor control after FSRT in a prospective cohort of benign anterior skull base meningiomas and pituitary adenomas.

\section{Methods}

\section{Study protocol}

Patients were recruited consecutively and prospectively into a local treatment protocol and database for anterior skull base meningiomas and pituitary adenomas during the period January 1999-December, 2009 at Rigshospitalet, Copenhagen, Denmark. This report includes patients 18 years of age and older at the time of treatment. Diagnosis was based on histological confirmation, or typical radiographic appearance for cases without surgery. Only patients with ophthalmological examinations and magnetic resonance imaging (MRI) scans at least 2 years after FSRT treatment were included in the analysis. Patients with malignant tumors were excluded.

\section{Patient population}

A total of 44 patients with anterior skull base meningiomas and 66 patients with pituitary adenomas were treated with FSRT. One patient diagnosed with a malignant meningioma was excluded. Of the remaining 43 patients with anterior skull base meningiomas, 2 patients died of unrelated causes within 2 years of treatment, 1 patient emigrated and further 1 patient was lost to follow-up, leaving 39 patients with $\geq 2$ years follow-up available for analysis. Of the 66 patients with pituitary adenomas, 2 patients died of unrelated causes within 2 years of treatment, 3 patients emigrate, and further 6 patients were lost to follow-up, leaving 55 patients with $\geq 2$ years follow-up available for analysis. For age and sex distribution, tumor characteristics, including tumor volume and details of surgical treatment, see Table 1.

Fractionated stereotactic radiation therapy

In all cases, a linear accelerator was used to deliver the stereotactic irradiation treatments; a Clinac 600SR (Varian Medical Systems) machine was used 1999-2008, and was thereafter replaced by 3 NovalisTx $^{\odot}$ machines (Varian Medical Systems, Palo Alto, CA, and BrainLab, München, Germany). Micro-Multi-leaf collimators were used since
Table 1 Patients, tumor characteristics and surgical treatment

\begin{tabular}{|c|c|}
\hline \multicolumn{2}{|l|}{ Anterior skull base meningiomas } \\
\hline \multicolumn{2}{|l|}{ Total 39} \\
\hline Females & $30(77 \%)$ \\
\hline Males & $9(23 \%)$ \\
\hline Mean age (years) & $56(37-78)$ \\
\hline \multicolumn{2}{|l|}{ Pituitary adenomas } \\
\hline Total & 55 \\
\hline Females & $29(53 \%)$ \\
\hline Males & $26(47 \%)$ \\
\hline Mean age (years) & $48(18-73)$ \\
\hline \multicolumn{2}{|c|}{ Anterior skull base meningiomas, tumor location } \\
\hline Cavernous sinus & $23(59 \%)$ \\
\hline Clival/Petroclival & $12(31 \%)$ \\
\hline Sphenoid wing & $3(8 \%)$ \\
\hline Optic canal & $1(2 \%)$ \\
\hline $\begin{array}{l}\text { Anterior skull base meningiomas, } \\
\text { tumor volume }\end{array}$ & $\begin{array}{l}21.02 \mathrm{cc}(\text { mean, } \\
\text { range } 0.33-152.50)\end{array}$ \\
\hline \multicolumn{2}{|l|}{ Pituitary adenomas, tumor extension } \\
\hline Intrasellar & $5(9 \%)$ \\
\hline Extrasellar & $50(91 \%)$ \\
\hline Pituitary adenoms, tumor volume & $\begin{array}{l}6.66 \mathrm{cc}(\text { mean } \\
\text { range } 0.00-41.83)\end{array}$ \\
\hline \multicolumn{2}{|l|}{ Pituitary adenomas, secretory status } \\
\hline Somatotrophic (GH secreting) & $34(62 \%)$ \\
\hline Corticotrophic (ACTH secreting) & $4(7 \%)$ \\
\hline Lactotrophic (prolactin secreting) & $2(4 \%)$ \\
\hline Null adenoma (non-secreting) & $15(27 \%)$ \\
\hline \multicolumn{2}{|l|}{ Operation prior to FSRT } \\
\hline \multicolumn{2}{|l|}{ Anterior skull base meningiomas } \\
\hline Total & $25(64 \%)$ \\
\hline One/two/three operations & $13 / 8 / 4$ \\
\hline $\begin{array}{l}\text { Median time from last operation } \\
\text { until FSRT: }\end{array}$ & 28 months (range $1-102$ ) \\
\hline \multicolumn{2}{|l|}{ Pituitary adenomas } \\
\hline Total & $49(91 \%)$ \\
\hline One/two/three/four operations & $24 / 16 / 9 / 1$ \\
\hline $\begin{array}{l}\text { Median time from last operation } \\
\text { until FSRT: }\end{array}$ & 10 months (range $1-134$ ) \\
\hline
\end{tabular}

2002, and fixed cones for the first patients. Initially, we used circular collimators, $0.5-3.5 \mathrm{~cm}$ diameter with $0.5 \mathrm{~cm}$ increments between sizes. From August 2000 and onwards, collimation and field shaping has been provided by micromultileaf collimators after upgrading. The treatment planning was performed using a system dedicated for stereotactic radiotherapy (XKnife, RSA, US, and BrainScan and iPlan, BrainLab, Munich, Germany). A pencil beam-based dose calculation algorithm was used for all patients from 2001 to 2002, earlier dosimetry were based on Clarkson integration. All cases were treated with multiple FSRT. An individual mask of the head was made during the planning 
process and was used during all the treatments. The mask was fixed to the board during the radiation, providing immobilization. A fusion of MR and computed tomography (CT) scans was used as a visual treatment plan, whereby the gross target volume (GTV) was estimated. The target volume was defined and treated without setup margins. The prescription dose of $54 \mathrm{~Gy}$ was typically prescribed to the $90 \%$ isodose contour, and the $90 \%$ isodose contour was encompassing the target volume. Prescribed tumor radiation doses were 1.8 Gy per dose given in 30 fractions or 2.0 Gy per dose given in 27 fractions (see also Table 2). Nineteen patients received treatment with intensity modified radiation therapy (IMRT) and 75 patients received dynamic three-dimensional Conformal Radiation Therapy (3DCRT arcs).

Visual pathways, definition and follow-up

Prospective ophthalmological follow-up after FSRT was scheduled at 9 months, 2 years, 3.5 years, 5 years, 7 years and 10 years after treatment. Visual acuity was measured with a Snellen eye chart and an improvement in visual acuity was defined as a gain of two or more Snellen lines, and worsening was defined as a loss of two or more lines. Visual fields were quantified with Campimetry, Goldmann dynamic perimetry and Octopus static perimetry. The central $30^{\circ}$ of the visual fields for each eye separately, were subdivided into an inner, middle and outer circle area, and each circle subdivided into 8 fields, resulting in a maximum of 24 defects per eye. An improvement in visual fields was defined as a gain of 1/24 or more and worsening was defined as a loss of $1 / 24$ or more of the fields for any eye.

Optic radiation dose and statistical analysis

The combined optic structure (COS) (optic nerves, chiasm and tracts) was outlined by BrainScan or BrainLab autocontouring function. The presence or absence of direct contact between tumor and anterior visual pathways was determined from the 3-dimensional dose plan MRI scans. Dosimetry data were extracted from the dose plan records. Most patients had only the COS delineated. A few patients had data separately for optic chiasm, tracts and nerves on the left and right sides (20 out of 94). For RION events analysis, dose-data were corrected to a radiobiologically equivalent dose in 2 Gy per fraction using the linear-quadratic model and an alpha/beta ratio of 3, which was used subsequently in the analysis. Further, only the maximum dose of the COS was considered, extracting the maximum of the dose delivered to the chiasm, optic nerves and optic tracts.
Table 2 Prescribed tumor treatment doses to the $90 \%$ isodose line, combined optic apparatus (COP) radiation doses (median of maximum doses) and proximity to the anterior visual pathways

\begin{tabular}{ll}
\hline Anterior skull base meningiomas & \\
Tumor dose $1.8 \times 30$ & $31(79 \%)$ \\
Tumor dose $2.0 \times 27$ & $8(21 \%)$ \\
COS (optic nerves + chiasm + tracts) & $53.3($ range 6.6-63.6) \\
Tumor-anterior visual pathways & $24(61.5 \%)$ \\
$\quad$ direct contact & \\
Pituitary adenomas & $35(64 \%)$ \\
Tumor dose $1.8 \times 30$ & $20(36 \%)$ \\
Tumor dose $2.0 \times 27$ & $54.6($ range $17.4-64.2)$ \\
COS (optic nerves + chiasm + tracts) & $12(22 \%)$ \\
Tumor-anterior visual pathways & \\
$\quad$ direct contact & \\
\hline
\end{tabular}

The visual outcome was categorized as RION visual acuity event in case acuity dropped from greater than $0.2-0.2$ or less on one or both eyes. Visual field decrease by $4.2,12.5,25$ and $50 \%$ or more $(1 / 24,3 / 24,6 / 24$ and $12 / 24$ fields) on one or both eyes, respectively was categorized as an RION visual field event. Patients with tumor growth or the occurrence of other optic lesion following FSRT were censored. We investigated if both RION field and acuity events were associated with maximum radiation dose to the COS, dose per fraction, number of operations performed pre-FSRT, age at FSRT, diagnosis and tumor size at baseline using Cox regression models. A $p$ value of 0.004 was considered significant (Bonferroni correction 0.05/14).

In a post hoc analysis we investigated the association of maximum radiation dose to $\mathrm{COS}$, treatment technique (IMRT or 3DCRT), tumor size and if the tumor was in direct contact with the optic nerves/chiasm (SPSS, IBM, US). Visual acuity and field function at baseline, categorized as "intact" or "impaired", was compared to the function following FSRT categorized as "improved", "unchanged" or "worsened". Patients with an "intact" status at baseline were by definition not considered able to "improve". The risk of having worsened function was compared for the groups where function was intact and impaired at baseline (Fisher's exact test, two-tailed) using the $\mathrm{R}$ statistical package ( $\mathrm{R}$ version 3.0.1, 2013-05-16). Also, the difference of visual acuity and fields function at baseline for meningioma and pituitary adenoma patients were compared (Fisher's exact test, two-tailed). In the post hoc analysis, a $p$-value of 0.05 was considered significant.

Tumor control measures

Prospective neuroimaging follow-up after FSRT was scheduled at 9 months, 2 years, 3.5 years, 5 years, 7 years 
Table 3 Visual function

\begin{tabular}{|c|c|c|c|c|c|}
\hline & Baseline status & Improved & Unchanged & Worsened & Fisher's exact test \\
\hline \multirow[t]{4}{*}{ Anterior skull base meningiomas } & Visual acuity intact & - & 18 & 1 & \multirow[t]{2}{*}{$p=1.000$} \\
\hline & Visual acuity impaired & 2 & 16 & 2 & \\
\hline & Visual fields intact & - & 21 & 1 & \multirow[t]{2}{*}{$p=0.068$} \\
\hline & Visual fields impaired & 3 & 9 & 5 & \\
\hline \multirow[t]{4}{*}{ Pituitary adenomas } & Visual acuity intact & - & 33 & 1 & \multirow[t]{2}{*}{$p=0.064$} \\
\hline & Visual acuity impaired & 2 & 15 & 4 & \\
\hline & Visual fields intact & - & 37 & 5 & \multirow[t]{2}{*}{$p=0.192$} \\
\hline & Visual fields impaired & 3 & 6 & 4 & \\
\hline
\end{tabular}

Fisher's exact test (two tailed). Comparing the fraction of patients ending up with an unchanged or improved versus worsened function, on last follow-up, who had an intact versus impaired visual function at baseline

and 10 years after treatment, with T1-weighted MRI images of the head, after application of Gadolinium, in the three planes in all studies. Tumor size was measured in three dimensions: antero-posterior, lateral and cranio-caudal. Largest tumor dimensions were defined on pre-treatment and the latest post-treatment contrast MRI studies. Tumor control was defined as regression or stable tumor size. Only a change of $2 \mathrm{~mm}$ or more in any dimension was considered a change in tumor size. Tumor volume in $\mathrm{cm}^{3}$ (cc) was defined using treatment planning software.

\section{Results}

Visual outcome

Median follow-up after FSRT for anterior skull base meningiomas was 5.4 years (range 2.7-10.6) and for pituitary adenomas 6.8 years (range 3.0-13.4). Visual acuity for anterior skull base meningiomas of the left eye was $0.86( \pm 0.36)$ pre- and $0.84( \pm 0.36)$ post-FSRT and of the right eye $0.77( \pm 0.39)$ pre- and $0.76( \pm 0.41)$ postFSRT. Visual acuity for pituitary adenomas of the left eye was $0.95( \pm 0.24)$ pre- and $0.95( \pm 0.28)$ post-FSRT and of the right eye $0.85( \pm 0.30)$ pre- and 0.87 ( \pm 0.33$)$ postFSRT. Visual baseline and outcome data are presented in Table 3. Baseline visual fields were significantly better for the pituitary adenoma group $(p=0.049)$, while visual acuity was not significantly different at baseline ( $p=0.291)$ for meningioma and pituitary adenoma patients. Meningioma patients with impaired visual fields at baseline had a slightly higher risk for worsened function following FSRT $(p=0.068)$. Pituitary adenoma patients with impaired visual acuity at baseline had a slightly higher risk for worsened function following FSRT $(p=0.064)$. Pooling the data for both diseases, the risk of worsened visual fields and visual acuity following FSRT, was elevated for patients with an impaired function at baseline ( $p=0.016,0.067$, respectively).
Radiation induced optic neuropathy (RION)s

Eighty-eight patients were evaluable with respect to RION after excluding six patients with pre-treatment blindness due to tumor compression (two patients), cataract, keratitis, optic neuritis and bilateral glaucoma. Radiation-induced visual symptoms were identified in 15 of the 94 patients in the whole series, including 11 patients with RION and 4 patients with lens or cornea damage. Of the 39 patients with anterior skull base meningiomas, 4 patients $(10.3 \%)$ developed new visual field defects after FSRT, at 6.1-25.5 months after FSRT, in the absence of other optic lesions or tumor growth, and were therefore considered to have RION. Of the 55 patients with pituitary adenomas, 7 patients $(12.7 \%)$ developed new visual field defects, and one of these also developed visual acuity loss, at 9.0-79.6 months after FSRT, in the absence of other optic lesions or tumor growth, and were therefore considered to have RION.

Optic radiation dose, tumor-optic direct contact and statistical analysis

Fourteen, 21 and 6 patients received a COS dose of 55-60, 60-65 and 65-68 Gy (2-Gy equivalent dose), respectively (see Table 2 for details). With only one RION acuity loss event observed, no meaningful statistical analysis could be performed for RION acuity loss. For the patient with RION acuity event, the maximum dose to COS was a modest 29 Gy. RION field loss of 1-2, 3-5, 6-11 and 12-24 fields out of 24 were observed for three, five, six and four eyes, respectively, out of the evaluable cohort.

Tumor was found to be in direct contact with the anterior visual pathways in $24(61.5 \%)$ cases of anterior skull base meningiomas and in $12(20 \%)$ cases of pituitary adenomas, with a variable degree of compression of the optic nerves or chiasm (Table 2). The association between tumor volume (cc), maximum COS dose (Gy), tumor-optic direct contact $(0=$ no, $1=$ yes $)$, IMRT use $(0=$ no, 


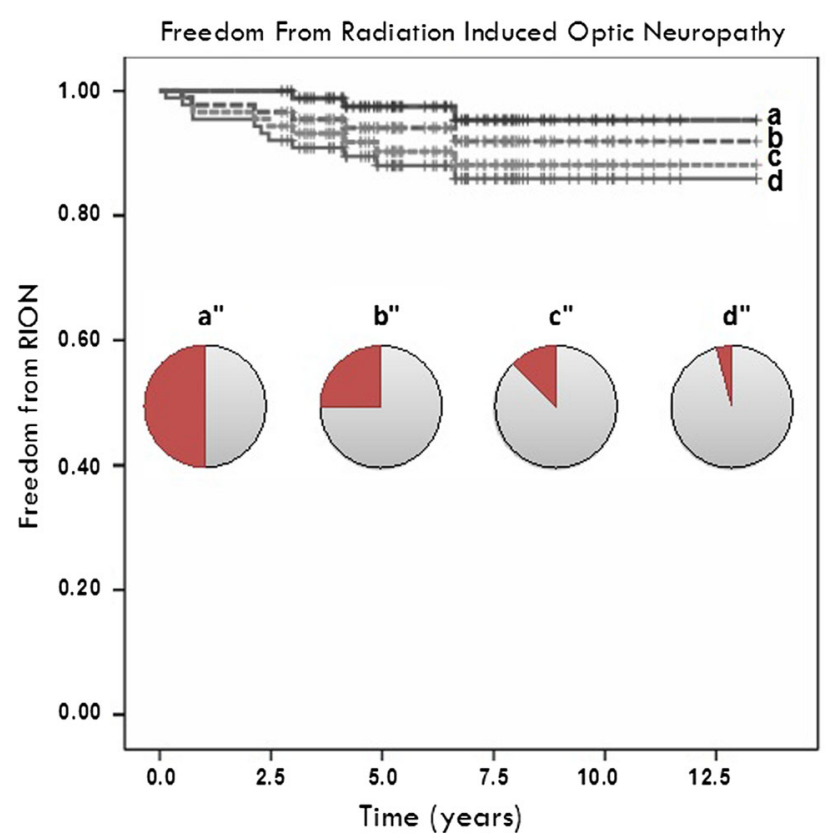

Fig. 1 Actuarial data for RION field loss for all patients, with patients having a loss of half or more, $\mathbf{b}$ a quarter or more, $\mathbf{c} 1 / 8$ th or more and d $1 / 24$ th or more of the visual field for one or both eyes

$1=$ yes) and disease (pituitary $=0$, meningioma $=1$ ) was investigated using Spearman signed rank test. COS maximum dose, was associated with tumor-optic direct contact $(\rho=0.312, p=0.002)$. Also, IMRT use resulted in lower doses to the $\operatorname{COS}(\rho=-0.415, p<0.001)$. Larger tumors indicated increased dose to the COS ( $\rho=0.236, p=0.022$ ) and were more likely to be associated with compression or distortion of the optical structures $(\rho=0.434, p<0.001)$. However, maximum dose to COS and tumor size at FSRT were non-significantly associated with RION field loss events. Also, age, diagnosis, dose per fraction $(2.0$ or $1.8 \mathrm{~Gy})$ and number of operations before radiation therapy were all non-significantly associated with RION field loss events. Actuarial data for RION field loss are shown in Fig. 1; 5-year Kaplan-Meier estimated freedom from $25 \%$ RION visual field loss was $94 \%$.

\section{Long-term tumor control after FSRT}

For anterior skull base meningiomas, median follow-up was 6.4 years (range 2.7-11.7). Actuarial 2-, 5- and 10-year tumor control rates post FSRT were 100, 88.4 (95\% CI 72.2-94.9) and 64.5\% (95\% CI 28.3-85.9), respectively (Fig. 2). Mean tumor volume pre-FSRT was $21.02 \mathrm{cc}$ (range 0.33-152.50) and post-FSRT $22.62 \mathrm{cc}$ (range 0.16-184.70). One patient (2.6\%) underwent reoperation 55 months after FSRT due to symptomatic tumor progression causing exophthalmos.

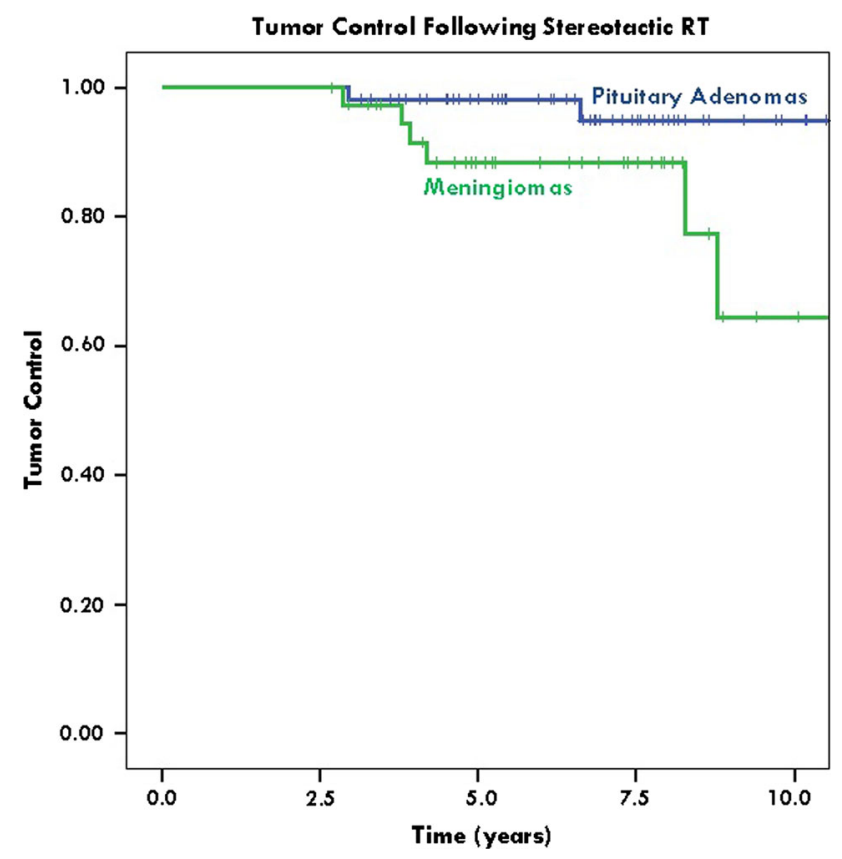

Fig. 2 Kaplan-Meier plots of long term tumor control, with $95 \%$ confidence interval lines. Actuarial 2-, 5- and 10-year tumor control rates post FSRT were 100, $88.4 \%$ (95\% CI 72.2-94.9) and $64.5 \%$ (95\% CI 28.3-85.9), respectively, for anterior skull base meningiomas, and 100, $98.2 \%$ (95\% CI 87.5-98.5) and $94.9 \%$ (95\% CI 79.8-98.5), respectively, for pituitary adenomas

For pituitary adenomas, median follow-up was 6.8 years (range 2.9-13.4 years). Actuarial 2-, 5- and 10-year tumor control post FSRT were 100, $98.2 \%$ (95 \% CI 87.5-98.5) and $94.9 \%$ (95 \% CI 79.8-98.5), respectively (Fig. 2). Mean tumor volume pre-FSRT was $6.66 \mathrm{cc}$ (range 0.00-41.83) and post FSRT $3.75 \mathrm{cc}$ (range 0.00-38.13). One patient $(2.0 \%)$ underwent reoperation 35 months after FSRT due to symptomatic tumor progression causing visual deterioration.

\section{Discussion}

This is, to our knowledge, the largest prospective study of the occurrence of RION after linear accelerator-based FSRT of anterior skull base meningiomas and pituitary adenomas. We found the occurrence of radiation-induced optic neuropathy after FSRT to be as high as $10 \%$ in patients with anterior skull base meningiomas and $13 \%$ with pituitary adenomas, although in the majority of most cases these were mild to moderate. Only one patient displayed marked visual acuity loss and worsening of visual fields was mild to moderate in most cases. Importantly, vision remained stable in a majority of patients and improved in a subset of patients. 
Our data show a lower occurrence of RION than reported by Emami [5], and support the view presented in the recent review by the QUANTEC initiative, reporting expected risks of 3-7 and 7-20\% of RION in the dose range 55-60 and above $60 \mathrm{~Gy}$, respectively, for maximum dose to the optic nerves [4]. A comparison with historical risk data is often complicated, though, and differences may be due to treatment planning, delivery aspects and clinical evaluations and other unknown factors.

In comparison to RION acuity loss, RION field loss was more prevalent, but appeared not to be associated with the maximum dose to the optic structures. Furthermore, although the radiation dose to the optic structures was higher for larger tumors and tumors compressing the optic structures, this increased dose was not associated with a difference in the rate of RION events. There might be several reasons for this lack of association. For example, MRI-tractography could be used for improved definition the optic pathways, but was not available for the present analysis. Also, minor positioning inaccuracies can cause rather substantial change in the maximum radiation dose due to the sharp dose-gradients of the treatment plans. Additionally, the dose-volume relationships are complex. A more in-depth analysis addressing these points is however beyond the scope of this work. SRS and FSRT both aim at sparing nearby eloquent structures, such as the optic chiasm [1]. Fractionated therapy has the biological advantage over single dose radiosurgery that it divides the total radiation dose into several smaller doses, so that a high radiation dose to the healthy structures in one single session is avoided [1]. In particular, SRS may not be suitable for lesions in close proximity to the visual pathways, due to a dose-limiting factor, although SRS studies have not been unequivocal, in particular regarding the dose-response tolerance of the optic pathways. For example, it was found that $<10$ Gy to lesions of the cavernous sinus in a single dose did not cause risk to the optic pathways, while 10-15 and $>15$ Gy caused a 27 and $78 \%$ incidence of optic neuropathy, respectively [6]. Other SRS studies of perioptic tumors have reported variable results. Two large Gamma Knife (GK)-SRS studies reported a 0 and $1.9 \%$ incidence of RION, respectively, at optic doses of $10 \mathrm{~Gy}[7,8]$, but another study reported a $4 \%$ incidence at an optic dose of 3, 1 Gy [9]. Large LINAC-SRS studies have reported a low incidence of RION of 0-2.8\% at optic doses less than 10 Gy [10-12]. Finally, a large combined LINAC/GK-SRS study found a $24 \%$ risk of RION at optic doses greater than $8 \mathrm{~Gy}$, but none at doses less than 8 Gy [13].

Our visual outcome data compare somewhat unfavorably with earlier series of tumors of the anterior skull base region treated with FSRT, with several studies reporting a low $0-6 \%$ incidence of visual loss for meningiomas around the anterior visual pathways [3, 14-20] and similarly for pituitary adenomas, in the range 0-9 \% [2, 21-29]. However, direct comparison with these previous studies is difficult, since median follow-up for most of them was significantly shorter [2, 3, 15, 16, 19, 20, 22, 24-27, 29] and several included a number of patients with less than 2 years follow up [2, 16, 20, 22, 24, 26, 27, 29, 30]. Indeed in our series, RION was in many cases a late event. More importantly, formal ophthalmological examinations were only consistently available in approximately half of the previous series [2, 17, 18, 20, 22-24, 26, 27, 31] and only a minority of the studies reported optic radiation dose $[2,17$, 18, 23-25].

More recently, Stiebel-Kalish and colleagues [32] reported 16 patients with meningiomas around the anterior visual pathways, treated with LINAC-FSRT, with regular ophthalmological examinations, and in line with our outcome data, found a $12 \%$ incidence of overall worsening of visual function. However, Kocher and colleagues, in another recent LINAC-FSRT series of 29 patients with perioptic tumors with a maximal total dose of 53.3 an 54.3 Gy to the optic nerves and chiasm, respectively, and maximal fractions of 1.86, reported only two new cases of disease related visual loss, but patients with short follow up were included [33].

In our study, tumor was found to be in direct contact with the anterior visual pathways in $24(61.5 \%)$ cases of anterior skull base meningiomas and in $12(20 \%)$ cases of pituitary adenomas and the optic apparatus as is often the case with these tumors, making it inevitable with current techniques that the optic apparatus often receives irradiation as high as the tumor itself. Similarly, Adler et al. [34] in a Cyberknife multisession radiosurgery series of perioptic tumors, all tumors were located within $2 \mathrm{~mm}$ of the optic apparatus, although they reported a high visual preservation of $94 \%$ of cases.

The 5 year tumor control rate of $88.4 \%$ for the anterior skull base meningiomas did not differ significantly from that of other FSRT series reporting a tumor control rate of $88-98 \%[14,16,30,32,35,36]$ and the 5 year tumor control rate for the pituitary adenomas of $98.2 \%$, compared favorably with the rates of 93-99\% reported in other FSRT series [21-24, 27]. Furthermore, although tumors close to the visual pathways treated with FSRT tend to be larger, our 5 syear tumor control rates seem to be comparable even with those reported for large LINAC-SRS [1012, 37] or GK-SRS series [7, 38, 39].

In conclusion, balanced against the risks of uncontrolled tumor growth it may be concluded that FSRT is a relatively safe and effective treatment of these tumors. Improved dose planning techniques may be able to reduce the incidence of visual complications further. 
Acknowledgments Special thanks to Tina Sommer and Tove Rømer for administrative assistance. The study was funded by The Capital Region of Denmark Research grant.

Conflict of interest The authors declare that they have no conflict of interest.

Ethical standards The study was conducted according to Danish legislation. Ethical committee approval is not required in Denmark for clinical audits.

Open Access This article is distributed under the terms of the Creative Commons Attribution License which permits any use, distribution, and reproduction in any medium, provided the original author(s) and the source are credited.

\section{References}

1. Roug S, Rasmussen AK, Juhler $M$ et al (2010) Fractionated stereotactic radiotherapy in patients with acromegaly: an interim single-centre audit. Eur J Endocrinol 162:685-694

2. Elhateer H, Muanza T, Roberge D et al (2008) Fractionated stereotactic radiotherapy in the treatment of pituitary macroadenomas. Curr Oncol 15:286-292

3. Litre CF, Colin P, Noudel R et al (2009) Fractionated stereotactic radiotherapy treatment of cavernous sinus meningiomas: a study of 100 cases. Int J Radiat Oncol Biol Phys 74:1012-1017

4. Mayo C, Martel MK, Marks LB, Flickinger J, Nam J, Kirkpatrick J (2010) Radiation dose-volume effects of optic nerves and chiasm. Int J Radiat Oncol Biol Phys 76:S28-S35

5. Emami B, Lyman J, Brown A et al (1991) Tolerance of normal tissue to therapeutic irradiation. Int J Radiat Oncol Biol Phys 21:109-122

6. Leber KA, Bergloff J, Pendl G (1998) Dose-response tolerance of the visual pathways and cranial nerves of the cavernous sinus to stereotactic radiosurgery. J Neurosurg 88:43-50

7. Morita A, Coffey RJ, Foote RL, Schiff D, Gorman D (1999) Risk of injury to cranial nerves after gamma knife radiosurgery for skull base meningiomas: experience in 88 patients. J Neurosurg 90:42-49

8. Stafford SL, Pollock BE, Leavitt JA et al (2003) A study on the radiation tolerance of the optic nerves and chiasm after stereotactic radiosurgery. Int J Radiat Oncol Biol Phys 55:1177-1181

9. Cifarelli CP, Schlesinger DJ, Sheehan JP (2012) Cranial nerve dysfunction following Gamma Knife surgery for pituitary adenomas: long-term incidence and risk factors. J Neurosurg 116:1304-1310

10. Spiegelmann R, Cohen ZR, Nissim O, Alezra D, Pfeffer R (2010) Cavernous sinus meningiomas: a large LINAC radiosurgery series. J Neuro oncol 98:195-202

11. Runge MJ, Maarouf M, Hunsche S et al (2012) LINAC-radiosurgery for nonsecreting pituitary adenomas. Long-term results. Strahlenther Onkol 188:319-325

12. Spiegelmann R, Nissim O, Menhel J, Alezra D, Pfeffer MR (2002) Linear accelerator radiosurgery for meningiomas in and around the cavernous sinus. Neurosurgery 51:1373-1379 discussion $9-80$

13. Tishler RB, Loeffler JS, Lunsford LD et al (1993) Tolerance of cranial nerves of the cavernous sinus to radiosurgery. Int J Radiat Oncol Biol Phys 27:215-221

14. Metellus P, Batra S, Karkar S et al (2010) Fractionated conformal radiotherapy in the management of cavernous sinus meningiomas: long-term functional outcome and tumor control at a single institution. Int J Radiat Oncol Biol Phys 78:836-843
15. Brell M, Villa S, Teixidor $P$ et al (2006) Fractionated stereotactic radiotherapy in the treatment of exclusive cavernous sinus meningioma: functional outcome, local control, and tolerance. Surg Neurol 65:28-33

16. Jalali R, Loughrey C, Baumert B et al (2002) High precision focused irradiation in the form of fractionated stereotactic conformal radiotherapy (SCRT) for benign meningiomas predominantly in the skull base location. Clin Oncol (R Coll Radiol) 14:103-109

17. Milker-Zabel S, Zabel A, Schulz-Ertner D, Schlegel W, Wannenmacher M, Debus J (2005) Fractionated stereotactic radiotherapy in patients with benign or atypical intracranial meningioma: long-term experience and prognostic factors. Int $\mathbf{J}$ Radiat Oncol Biol Phys 61:809-816

18. Milker-Zabel S, Zabel-du Bois A, Huber P, Schlegel W, Debus J (2006) Fractionated stereotactic radiation therapy in the management of benign cavernous sinus meningiomas : long-term experience and review of the literature. Strahlenther Onkol 182:635-640

19. Pirzkall A, Debus J, Haering P et al (2003) Intensity modulated radiotherapy (IMRT) for recurrent, residual, or untreated skullbase meningiomas: preliminary clinical experience. Int J Radiat Oncol Biol Phys 55:362-372

20. Milker-Zabel S, Zabel-du Bois A, Huber P, Schlegel W, Debus J (2007) Intensity-modulated radiotherapy for complex-shaped meningioma of the skull base: long-term experience of a single institution. Int J Radiat Oncol Biol Phys 68:858-863

21. Colin P, Jovenin N, Delemer B et al (2005) Treatment of pituitary adenomas by fractionated stereotactic radiotherapy: a prospective study of 110 patients. Int J Radiat Oncol Biol Phys 62:333-341

22. Paek SH, Downes MB, Bednarz G et al (2005) Integration of surgery with fractionated stereotactic radiotherapy for treatment of nonfunctioning pituitary macroadenomas. Int J Radiat Oncol Biol Phys 61:795-808

23. Weber DC, Momjian S, Pralong FP, Meyer P, Villemure JG, Pica A (2011) Adjuvant or radical fractionated stereotactic radiotherapy for patients with pituitary functional and nonfunctional macroadenoma. Radiat Oncol 6:169

24. Milker-Zabel S, Debus J, Thilmann C, Schlegel W, Wannenmacher M (2001) Fractionated stereotactically guided radiotherapy and radiosurgery in the treatment of functional and nonfunctional adenomas of the pituitary gland. Int J Radiat Oncol Biol Phys 50:1279-1286

25. Mitsumori M, Shrieve DC, Alexander E 3rd et al (1998) Initial clinical results of LINAC-based stereotactic radiosurgery and stereotactic radiotherapy for pituitary adenomas. Int J Radiat Oncol Biol Phys 42:573-580

26. Jalali R, Brada M, Perks JR et al (2000) Stereotactic conformal radiotherapy for pituitary adenomas: technique and preliminary experience. Clin Endocrinol (Oxf) 52:695-702

27. Minniti G, Traish D, Ashley S, Gonsalves A, Brada M (2006) Fractionated stereotactic conformal radiotherapy for secreting and nonsecreting pituitary adenomas. Clin Endocrinol (Oxf) 64:542-548

28. Snead FE, Amdur RJ, Morris CG, Mendenhall WM (2008) Longterm outcomes of radiotherapy for pituitary adenomas. Int $\mathbf{J}$ Radiat Oncol Biol Phys 71:994-998

29. Sun DQ, Cheng JJ, Frazier JL et al (2010) Treatment of pituitary adenomas using radiosurgery and radiotherapy: a single center experience and review of literature. Neurosurg Rev 34:181-189

30. Minniti G, Clarke E, Cavallo L et al (2011) Fractionated stereotactic conformal radiotherapy for large benign skull base meningiomas. Radiat Oncol 6:36

31. Behbehani RS, McElveen T, Sergott RC, Andrews DW, Savino PJ (2005) Fractionated stereotactic radiotherapy for parasellar meningiomas: a preliminary report of visual outcomes. $\mathrm{Br} \mathrm{J}$ Ophthalmol 89:130-133 
32. Stiebel-Kalish H, Reich E, Gal L et al (2012) Visual outcome in meningiomas around anterior visual pathways treated with linear accelerator fractionated stereotactic radiotherapy. Int J Radiat Oncol Biol Phys 82:779-788

33. Kocher M, Treuer H, Hoevels M, Semrau R, Sturm V, Mueller RP (2013) Endocrine and visual function after fractionated stereotactic radiotherapy of perioptic tumors. Strahlenther Onkol 189:137-141

34. Adler JR Jr, Gibbs IC, Puataweepong P, Chang SD (2008) Visual field preservation after multisession cyber knife radiosurgery for perioptic lesions. Neurosurgery 62(Suppl 2):733-743

35. Hamm K, Henzel M, Gross MW, Surber G, Kleinert G, Engenhart-Cabillic R (2008) Radiosurgery/stereotactic radiotherapy in the therapeutical concept for skull base meningiomas. Zentralbl Neurochir 69:14-21
36. Henzel M, Gross MW, Hamm K et al (2006) Stereotactic radiotherapy of meningiomas: symptomatology, acute and late toxicity. Strahlenther Onkol 182:382-388

37. Jensen RL, Jensen PR, Shrieve AF, Hazard L, Shrieve DC (2010) Overall and progression-free survival and visual and endocrine outcomes for patients with parasellar lesions treated with intensity-modulated stereotactic radiosurgery. J Neuro oncol 98:221-231

38. Park KJ, Kano H, Parry PV et al (2011) Long-term outcomes after gamma knife stereotactic radiosurgery for nonfunctional pituitary adenomas. Neurosurgery 69:1188-1199

39. Pollock BE, Cochran J, Natt N et al (2008) Gamma knife radiosurgery for patients with nonfunctioning pituitary adenomas: results from a 15-year experience. Int J Radiat Oncol Biol Phys 70:1325-1329 